ORIGINAL ARTICLES

\title{
Perceptions and experiences of pregnant women towards voluntary antenatal counselling and testing in Oshakati hospital, Namibia
}

\author{
Aini-Kaarin Toivo ${ }^{1}$, Mickey Chopra ${ }^{2}$, Daniel Opotamutale Ashipala*3 \\ ${ }^{1}$ Ministry of Health and Social Services, Directorate of Primary Health Care Services, Family Health Division, Oshakati, Namibia \\ ${ }^{2}$ School of Public Health, Faculty of Community and Health, University of the Western Cape, Cape Town, South Africa \\ ${ }^{3}$ Department of General Nursing Science, School of Nursing, Faculty of Health Sciences, University of Namibia (UNAM), Rundu, \\ Namibia
}

Received: November 21, 2016

DOI: $10.5430 /$ ijh.v3n2p20
Accepted: June 19, 2017

Online Published: July 10, 2017

URL: https://doi.org/10.5430/ijh.v3n2p20

\begin{abstract}
Background: The study focused on perceptions and experiences of pregnant women who opted in against those who opted out of voluntary antenatal HIV counseling and testing.

Purpose: The purpose of the study was to assess pregnant women's perceptions and experiences in order to gain insight into their views towards voluntary antenatal counseling and testing.

Method: A qualitative, descriptive and comparative study was used regarding participants who opted in and opted out. A stratified random sampling was used to collect data from the participants. The study sample comprised six focus group discussions (FGD) of pregnant women who were attending antenatal services at this hospital. Three FGD were women who opted in and other three FGD were for those who opted out. The semi-structure interview guide was used to guide the discussions. The discussions were audio recorded, transcribed and analyzed, using thematic content analysis.

Results: Based on the findings of the study, the knowledge of pregnant women who opted in was relatively good comparing with those who opted out. Despite the recognition of the benefits of Voluntary antenatal Counselling and Testing (VCT), it was evident that women were reluctant to use the VCT service because of reasons that include fear of being stigmatized, abandoned and discriminated against. The women's participation in the existing services of voluntary antenatal counseling and testing was influenced by their partners. It was also found that there were misconceptions surrounding the knowledge of the causes and prevention of vertical transmission. These misconceptions should be talked about. The distance was another factor which hampered the participation of pregnant women in the programme. Other reasons for non-attendance given by the participants included dissatisfaction with some of the nurses' attitudes, ignorance about the service as well as reluctance to be tested.

Recommendations: This study recommends improved partner communication on the issues of HIV/AIDS as it will encourage sharing of confidentiality and responsibility.
\end{abstract}

Key Words: Perceptions, Experiences, Confidentiality, Uptake of HIV, Opt out testing, Opt in testing

*Correspondence: Daniel Opotamutale Ashipala; Email: dashipala@unam.na; Address: School of Nursing, Faculty of Health Sciences, University of Namibia (UNAM), Rundu, Kaisosi Road, Namibia. 


\section{INTRODUCTION}

HIV/AIDS has become the most significant public health problem worldwide. In 2014 the global epidemic killed more than 3 million people, of whom 2.3 million were in subSaharan Africa. ${ }^{[1]}$ The largest source of infection is HIV transmission from infected mother-to the-child. Scientific data now confirm that HIV can be transmitted from an infected mother to her child in the utero, during delivery and through breastfeeding. ${ }^{[2]}$ The Ministry of Health and Social Services (MOHSS) report (2014) revealed that one in five pregnant women is HIV infected in Southern African countries.

In Namibia, the first four cases of HIV were reported in 1986. Since that year, the HIV/AIDS cases have increased rapidly and become the leading cause of death from 1996. AIDS was responsible for $26 \%$ of all reported deaths, and for $46 \%$ of deaths in the $15-49$ year old group in $1999 .^{[1]}$ The number of HIV deaths has increased up to $80 \%$ in the three preceding years. ${ }^{[3]}$ By 31 December 2014, the accumulative number of HIV/AIDS cases, 136,068 was reported by the. ${ }^{[3]}$ In Oshakati, HIV infection among pregnant women was 18 $2 \%$ according to sentinel-sero survey for $2014 .^{[1]}$

It is against this background that voluntary HIV counselling and testing should be available and accessible to all pregnant women, in order to reduce the vertical transmission. Currently, most pregnant women do not know their HIV status. ${ }^{[2]}$ The reason behind is that they fear to receive positive results. It is now high time to promote, provide and improve the access to Voluntary antenatal Counselling and Testing (VCT) in areas where MTCT interventions are planned.

The Ministry of Health and Social Services in Namibia introduced the Prevention of mother to child HIV transmission (PMTCT) programme in 2002. It was piloted in two hospitals, Oshakati and Katutura. Since the implementation of this programme, the uptake has not increased as it was expected From 1 March 2002 - September 2002, about 1834 pregnant women attended the ANC for the first time at Oshakati Hospital. The numbers of pregnant women who enrolled for the programme and agreed to be tested during that period was considerably less, $462(25,2 \%){ }^{[1]}$

It is because of these reasons that the study was conducted, to compare the perceptions and experiences of pregnant women towards voluntary antenatal HIV counselling and testing. The results will be hopefully used to improve the implementation of VCT among pregnant women in the Oshakati District.

\subsection{Problem statement}

Statistics obtained from the records of the ANC in Oshakati Hospital showed that only $21 \%$ of HIV positive pregnant

Published by Sciedu Press women were identified in the programme. Between April to September 2002, 520 pregnant women visited the ANC for the first time and only $179(43.42 \%)$ pregnant women agreed to be tested. Out of that number $149(83,24 \%)$ pregnant women tested HIV positive while $30(16,75 \%)$ tested HIV negative and $1(0,22 \%)$ pregnant woman was doubtful. In 2003, 790 women visited the antenatal clinic for the first time but only $163(20,63 \%)$ pregnant women agreed to be tested. The total number of $99(60,74 \%)$ pregnant women enrolled tested HIV positive while $64(39,26 \%)$ pregnant women tested negative. The involvement of partners in the VCT (voluntary counseling and testing) is considerably low. The data shows that from March 2002 - September 2003, 35 $(11,71 \%)$ male partners enrolled in the programme. However, out of 35 male partners tested, only $31(88,5 \%)$ came back for post-test counseling Government of the Republic of Namibia. ${ }^{[1]}$

From the above statistics, it can be assumed that voluntary HIV counseling and testing services are under-utilized. In spite of the prevailing opportunity, i.e., high ANC attendance and hospital deliveries, the uptake of the programme has been low. This can also create an assumption that women have negative attitudes towards VCT during pregnancy. Therefore, the researchers were interested in the perceptions and experiences of pregnant women towards antenatal VCT. So far, no study has been conducted on the perceptions of pregnant women towards voluntary antenatal counseling and testing among pregnant women in Namibia. Therefore, the study results will be used as baseline information which will assist MOHSS to develop amicable strategies, which will improve the uptake of voluntary antenatal counseling and testing among pregnant women.

\subsection{Purpose of the study}

The purpose of the study was to compare the perceptions and experiences of pregnant women towards voluntary antenatal HIV counselling and testing in Oshakati Hospital in Namibia.

\subsection{Objectives and aim of the study}

\subsubsection{Aim}

The aim of the study was to compare the knowledge, attitude, experiences and beliefs towards voluntary HIV counseling and testing among pregnant women who opt in against those who opt out of the voluntary antenatal HIV counseling and testing.

\subsubsection{Objectives}

- To identify groups of pregnant women who opt in and those who opt out regarding voluntary antenatal HIV counseling and testing. 
- To compare knowledge about vertical transmission of HIV and voluntary antenatal HIV counseling and testing among pregnant women who opt in against those who opt out of the voluntary antenatal HIV counseling and testing.

- To compare the perceptions, attitudes, beliefs and experiences of pregnant women who opted in and opted out of voluntary antenatal HIV counseling and testing.

\section{STUDY DESIGN AND RESEARCH METHODS}

A comparative descriptive study design was utilized to conduct this study. It allowed the researcher to compare the perceptions and experiences regarding voluntary antenatal HIV counseling and testing between the pregnant women who opted in and those opted out.

The study was of qualitative nature. Qualitative research designs "focus on qualitative aspects (meaning, experience and understanding from the new point of the research subjects and in the context in which the acting takes place". ${ }^{[4]}$ Therefore the study was aimed to gain in-depth information by testing pregnant women's understanding, views, attitudes, and experiences towards voluntary antenatal HIV counseling and testing. "Qualitative research approach values participants perspectives and seek to discover these perspectives". ${ }^{[5]}$

\subsection{Study population}

Brink $^{[4]}$ defined study population as the entire groups or persons or objects that are of interest to the researcher. The study population was the pregnant women attending antenatal services at Oshakati Hospital ANC clinic, and who visited this health facility during the period of the study, September 2004. Therefore, Oshakati hospital antenatal clinic was selected as the area of the study.

\section{Sampling}

A stratified random sampling was used to select the participants. The study sample was drawn from pregnant women who are attending antenatal services at this hospital between the ages of 15-45. The study sample comprised 6 focus group discussions (FGD) of pregnant women who opted in and those who opted out. The stratum was pregnant women who opted in and opted out. Since there is already a system in place which is registering pregnant women, those who opt in and out, that register was used to select the participants. The nurses at ANC clinic assisted in selecting the potential participants for the proposed study. The pregnant women were randomly selected, by giving them numbers and every third pregnant women were selected. The stratified random sampling provides a representation of a particular segment of the population as suggested by Brink. ${ }^{[4]}$

\subsection{Data collection}

Data were collected from the 13-30 September 2004 in Oshakati Hospital ANC clinic. The focus group discussions were conducted within the period of three weeks, 13-30 September 2004. All the participants met the inclusion criteria: pregnant women who opt in and opt out, attending antenatal services in Oshakati Hospital ANC clinic. Six focus group discussions were conducted. The participants consisted of 36 pregnant women: 18 were pregnant women who opted in and 18 were pregnant women who opted out. Of the 36 participants 16 were married and 20 were not married.

To collect data, FGD interview guide was used as an instrument. A focus group is defined by Krueger ${ }^{[5]}$ as a carefully planned discussion designed to obtain perceptions of a defined area of interest in a permissive, threatening environment. The key principle in the formation of the focus group is homogeneity which is determined by the purpose of the study. Each FGD, which lasted for at least one hour, was used to compare perceptions, attitudes, beliefs and experiences of pregnant women who opted in and opted out of voluntary antenatal HIV counseling and testing. 3 FGD were for women who opted in and the 3 other FGD were for those who opted out and each FGD comprises of 10 pregnant women. The aim of FGD was to produce the thick in-depth information on perceptions, attitude, beliefs and experiences towards VCT. Pregnant women shared their experiences and how they perceived VCT. The researchers asked/posed probing questions until saturation of information was reached.

\subsection{Data analysis}

Data analysis started immediately after the interviews were completed, the data were analyzed. Thematic content analysis was used to analyze the data. Content analysis is the process of identifying, coding and categorizing the primary patterns in the data. ${ }^{[6]}$ The taped information was listened to and transcribed. The transcriptions were read carefully, jotting down ideas as they came in mind. A list of all topics was made and similar topics were clustered together to form categories. The topics were turned into categories and subcategories. The results of two groups were compared and analyzed. The systematic comparison during discussions between two groups on all topics was done.

\section{Trustworthiness of the study}

Rigour was ensured through credibility, transferability, dependability and confirmability. ${ }^{[7]}$ The researcher tried to adhere to the principles of trustworthiness throughout the research process. Trustworthiness is the method of ensuring rigour in qualitative research. ${ }^{[8]}$ To ensure credibility, the researcher built trust by explaining to the participants the 
aim of the research, utilization and dissemination of the information and its use. The researcher kept the daily notes and included the non-verbal reactions of the participants.

\subsection{Ethical considerations}

Ethical clearance was obtained from the University of Western Cape Ethical Committee and permission to proceed while approval was given by the Ministry of Health in Namibia. The study was explained to the participants and each participant gave consent.

\section{RESULTS AND DISCUSSIONS}

\subsection{Knowledge about vertical transmission}

\subsubsection{Causes of vertical transmission}

Generally the participants who opted in were aware that vertical transmission is transmitted through three main ways: during pregnancy, during delivery and through breast milk. They had demonstrated clear knowledge that HIV is transmitted through breast milk. They indicated that there were two feeding options which the pregnant women would consider if they were HIV positive: exclusive breast feeding for the first four months or formula feeding. They felt that those who could afford to buy formula milk could use formula feeding, since breast milk contained HIV. The following statement confirms:

"The nurses informed us that women should exclusively breastfeed, and no water must be given to the baby until it is four months old. After that women should stop breastfeeding and start giving the baby other types of food."

The participants who opted in knew that vertical transmission can be caused through unprotected sexual intercourse. They discouraged the pregnant women not to have sexual intercourse during pregnancy until they stopped breastfeeding. They said:

"If a pregnant woman is practicing unprotected sex, she can transmit HIV to her baby. It is good if she does not have sex during pregnancy and while she breastfeeds in order to protect her baby. The men are not reliable."

The majority of participants who opted in felt strongly that if a woman had sores on her nipples she could infect her baby. This is an indication that they had knowledge about the causes of vertical transmission.

"I am very sure that if you are breastfeeding your baby and you have sores on your nipples - then the child is sucking the blood coming from the nipples, which can cause HIV, you can infect your baby."

The participants who opted out were aware that vertical transmission is caused from mother to child. They were not spe- cific or knew more causes of vertical transmission like those who opted in. They only knew that a mother can infect her baby through breast milk. This was evident in the following account:

"The HIV infected mother who breastfeed the baby can infect her child. It is better to give soft porridge, 'etete' (thin porridge) or formula milk".

Even though the participants who opted in demonstrated the knowledge about causes of vertical transmission, there were misconceptions surrounding its causes. Like those who opted out some participants who opted in also believed that a mother and baby shared the same blood.

"The mother can infect the baby with HIV because the mother and the baby share the same blood. Infection can also take place at birth, when the umbilical cord is cut."

Some participants who opted out believed that if the mother had sores on the mouth or all over the body, she could infect her baby.

"If the mother has sores on the mouth or all over her body, she can infect her baby."

\subsubsection{Prevention of vertical transmission}

The participants who opted in were aware that vertical transmission can be prevented if pregnant women, whose blood tested HIV positive, receive antiretroviral drugs during the onset of labour and the baby is also given after delivery. They felt that having unprotected sex with an infected man while breastfeeding may lead to HIV transmission to the mother, who in turn can infect the baby.

"Oh yes, there are drugs available to prevent the virus not to transmit from the mother to the baby. The HIV positive pregnant women will get drugs that prevent the transmission."

The participants who opted out said that vertical transmission could be prevented, if pregnant women take ARV drugs. However they did not know when to take them. This shows that the information obtained was inadequate and unclear. They heard about the PMTCT messages, but it could be that messages were not clear to them. The following were the examples:

"You can get ARV drugs when you are pregnant and continue taking them for 6 weeks after delivery." "The drugs will also prolong the lives of the pregnant women."

Interestingly, participants who opted out mentioned that some traditional practices should be avoided in order to prevent HIV transmission from the mother to the baby. They gave an example:

"Sometimes, when a child is sick, it is believed that the mother 
is the cause. As a result, a small part is cut off the vagina of the mother and the blood is given to the child to lick. They put some blood on the hands of the baby. This practice should be discouraged."

Interestingly, both groups were equally knowledgeable that mixed feeding is likely to transmit HIV to the baby after birth. They prefer HIV positive women to choose between the following two feeding options: exclusive breastfeeding for the first four months and after that the mother can stop breast feeding, and introduce weaning feeding or formula feeding in order to prevent transmission of HIV after delivery. The following statements are accounts of this:

"But I heard that you should only give bottle milk or breast milk, if you mix food there is a big chance of infecting the baby." "What is really good is that mothers should not give any other food, only breastmilk in order to prevent vertical transmission."

\subsection{Knowledge about voluntary antenatal HIV coun- selling and testing \\ 3.2.1 Understanding of voluntary antenatal HIV coun- selling and testing}

The participants who opted in had good understanding of voluntary antenatal HIV counselling and testing. They described it as the voluntary willingness of pregnant women to go for HIV test during pregnancy without being forced.

"It is when a pregnant woman decides to go for the test on a voluntary basis and nobody is forcing her to take the test."

\subsubsection{Awareness of availability of VCT}

The participants who opted in knew that voluntary antenatal HIV counselling and testing was available at some selected health facilities. They knew of many locations where to get an HIV test, with almost all of them mentioning a health facility and others such as new start centres, youth centres and private doctors.

"You can go to the big hospitals, because smaller hospitals are not doing it. Private Doctors, youth centres and New Start Centre also offer this service."

In contrast, the majority of the participants who opted out mentioned that they were not aware of the availability of this service. They did not hear or had no idea about the existence of the voluntary antenatal counselling and testing service until they were informed when they started antenatal services. They further explained that they only knew that pregnant women would be tested for three specimens such as blood group, haemoglobin and syphilis.

"Yes I know that a pregnant woman can only be tested for the three bloods things: blood group, haemoglobin and for syphilis. But, the day I started the ANC, it was the first time I heard that they would test us for HIV on a voluntary basis."

\subsubsection{Sources of information}

The group who opted in received information regarding voluntary antenatal HIV counselling and testing from the hospitals/clinics, radio, TV, community meetings done by homecare givers and booklets. These were reflected by the respondents as follows:

"I heard about voluntary antenatal counselling and testing over the radio, hospitals and from the people from Catholic Aids Action and TKMOAMS who are doing house to house visits."

The participants who opted out seemed to have little source of information. They just heard about it when they started with ANC. Only few of them mentioned that they heard the message from the radio.

\subsubsection{Benefits of voluntary antenatal HIV counselling and testing}

The participants who opted out knew the benefits of voluntary antenatal HIV counselling and testing. Overall, these participants felt that the voluntary antenatal HIV counselling and testing was beneficial. It also appears that participants who opted in had more information than those who opted out. Their opinions are described below:

"It is good to be tested. I can be able to know my HIV status and I can have a healthy baby even if I am HIV positive, because I can get Nevirapine that will protect my baby not to be infected."

"I can start using a condom with my partner, especially if I am negative and he is positive. My husband might sleep around with other women, if I know my status; I will not sleep with him without using a condom."

Although the participants who opted out knew that it is good to be tested during pregnancy in order to save the lives of their babies, they felt that they would not do that. They felt that they were not yet ready to receive their results because it would cause physical and emotional abuse by their husbands and families. They stated:

"It is good to know where you are standing so that you can receive drugs that help the baby. I am not ready to go for the HIV test."

\subsection{Attitude towards voluntary antenatal HIV coun- selling and testing}

\subsubsection{Reasons for acceptance of HIV test}

Participants who opted in indicated that one of the reasons of accepting the HIV test was that they wanted to protect 
themselves and the lives of their babies. They wanted to have healthy babies and to receive treatment to prolong their lives.

"I can have a healthy baby even if I am HIV positive".

\subsubsection{Protect the mother and the baby}

The participants who opted out knew that the baby's life would be protected. They thought that the drugs that the pregnant women received at the onset of labour would prolong their lives. However, the information was not clear to them, whether they would also benefit from this programme. For example:

"The drugs which the pregnant women received during the onset on labour will help the baby not to be infected and prolong the life of the mother."

It is clear that the women who opted out had inadequate information. They seemed to mix information on drugs that prevent vertical transmission and those that prolong their lives (HAART). It is very necessary for women to be informed about all the benefits regarding PMTCT. The benefits are: reducing the vertical transmission, changing sexual behaviour, receiving HAART; and preventing infections and re-infections.

\subsubsection{Receive treatment}

It was very interesting to note that only the group who opted in demonstrated that they wanted to know their status and if they tested positive they would also be referred to the Communicable disease clinic (CDC) and receive antiretroviral treatment in order to prolong their lives. They knew that HIV is not curable; but lives can be prolonged. They explained:

"It is good. I know that it will not cure me but I can be able to know my HIV status in order to start treatment for HIV to prolong my life."

\subsubsection{Behavioural change}

Some participants who opted in indicated that behavioural change was also one of the factors that motivate people to go for HIV test in order to make correct decisions.

"If I know my status, I will not sleep with my husband without using a condom especially if I am negative and he is positive. Men are naughty. My husband can sleep around with other women without using a condom and when he comes home he would do it without a condom."

"It will help me to take correct decisions. If I tested positive, I will not have sex anymore because of re-infection. If the test is negative, I will tell my husband to change his behaviour not to be infected."

The participants who opted out mentioned that one of the reasons which could motivate pregnant women to take the

Published by Sciedu Press test was that they would do it out of concern about their own health and previous sexual behaviours.

"If a woman is concerned because she had unprotected sex she may think of going to take the test. A person can go for the HIV test if she realized that her health is going down."

Some participants who opted out expressed their feelings that they could not take the test because they knew that if they tested HIV positive, they would not be able to discuss safe sex or introduce condom use to their partners.

"I cannot tell my husband to use a condom although I know that he might be infected with the HIV virus, otherwise he will think that I have affairs with other men."

\subsubsection{Reasons for declining of HIV test}

The participants who opted out agreed that they could not take the test because they were afraid of their husbands who might hear about their results.

"My husband is intimidating me! For example one day I told him of my intention to go for the test. He said: "If you go there (hospital) and tested positive, you should not come back home. If you come here, I will kill you and then kill myself."

"Mhnn! We fear to be blamed by our husbands and their families. I will also be scared to tell my husband, because he will not accept the test. After delivery he may not name the child. If you go for the test you must expect everything, life or death. Men are crazy. They can even kill you and then kill themselves."

The participants who opted out felt that they could not tell their husbands; otherwise the story would spread to other people especially to their family members. They preferred not to tell them to avoid inconveniencies such as physical violence and also bad words from the husband's relatives. They mentioned the following:

"I told my husband once that I would go for the HIV test. He said that he would kill me if I went for the test. Therefore I did not go because I know that he will kill me."

However, the majority of participants who opted in had expressed confidence and trust in their husbands that they would support them and keep test results secret. They knew that their husbands would understand the situation. Some had already discussed with their partners to be tested and permission to go ahead was granted.

"I will tell my husband because he is the only one I trusting my life. In my case I discussed it with him and he agreed."

However, some participants who opted in found it difficult to negotiate the HIV test with their husbands because they 
did not know how they would react. On the other hand they felt that it was still good to know their status in order to make correct decisions. They also felt that it was their right. Those who discussed with their partners did not experience any violence. Their partners allowed them to go for the test. Even the pregnant single women who opted in indicated that they would ask for permission, first from their partners.

"Ahaa! You must be careful. Informing your husband is not easy, you should expect everything life or death." That is why you hear many women being killed by their boyfriends because they disclosed their status to them, but I felt that it is my right to know my status in order to make right decisions."

\subsubsection{Attitude of health workers}

It was very interesting to note that only participants who opted out mentioned that, one of the main reasons for not accepting the test during pregnancy was because of the attitude of health workers. They described the nurses as rude, who did not keep people's results confidential. They were not supportive. They explained the attitudes of health workers as follows:

"Sometimes people are scared because some nurses are shouting at us. They gossip also, and tell people in the community about our results."

"Counseling sometimes is not done properly. If a nurse is informing you about positive results, you start crying, but she would say, 'I am not the one who gave you the virus'. They are not supporting us."

"Yes, some nurses do talk. The story will spread. They do gossip and tell people in the community about the results". They added also: "If the counselors do not talk, where do the rumours come from?"

Some participants who opted out further explained that even if women did not reveal their status, people could also suspect them, if they see them sitting at the PMTCT clinic. They would start observing whether their mood would be normal or not. Mood normally changes because of the concerns and worries. They explained:

"If people see you going into room 6 they will start suspecting that you are HIV positive.”They will just observe your mood to see whether it has changed or not. If it changes then that will be the sign that you are HIV positive."

The majority of participants who opted in were confident that nobody would find out unless they disclosed their status themselves. They expressed their satisfaction with the attitudes of the health workers. They were confident that their results would be kept confidential and nothing would be leaking from the PMTCT clinic. They further explained that the nurses would give them support even after their results were made known, especially when tested HIV positive.

"I do not think that the people will find out. In the counselling office you are there only with the counsellor, they will not find out. They do keep information confidential". "Yes, if my results are good then I will be happy and tell everybody, that I am AIDS free, but if my results are bad I will not tell anybody. The mood will change. I will blame myself and won't even talk to them. The people will start then suspecting something."

\subsubsection{Fear of death and to be known as HIV positive}

Some pregnant women who opted out had a problem regarding the test, because they were scared of the positive result. They revealed that it would bring death nearer. They also did not want to be known as the people living with HIV/AIDS. Therefore, for them it was better not to be tested in order to avoid stress or even provoke it. They explained:

"It is bad if a person does not accept the results. She will become more concerned, worried and she will die soon because of the stress". It will probably raise many thoughts, especially if you see AIDS patients suffering in the hospital. It is not good. They are suffering a lot. The pain is the problem. I cannot imagine undergoing the same problems of pain. It makes me mad and confused."

"I don't care about the test. 'Tashiya ka Shilondelwa. Shoka tashi ya nashiye' (If something is coming, it does not need provocation. It will come). I came in the world to go, not to stay. This disease has come to kill people not animals ...(laughing).”

In contrast, during the discussions the participants who opted out had concern about their children who would be left behind. They did not trust their husbands with a responsibility of taking care of the children. They preferred not to go for the test in order to remain oblivious of their HIV status.

"People are afraid of death, especially when they have children. They are asking themselves: Who is going to take care of my children when I am gone? You know that men don't care about children." "We women are born to take care of the children, unlike men. These are the things that we are concerned about".

\subsubsection{Committing suicide}

It was very interesting to note that among both groups, who opted out and opted in, some women had similar feelings that if their results were positive they would kill themselves. They further stated that to be HIV positive, one was almost dead and there was no future. This is evident from the following accounts: 
"Fear to be tested HIV positive. After the test you will not be the same". You will start thinking about many things. If the result is HIV positive, then I will kill myself. Especially when you look at people who are sick, they are really suffering. One can just decide to kill herself."

\subsection{Confidentiality, stigma and discrimination 3.4.1 Disclosure of HIV results}

The majority of the participants who opted in were confident that they would disclose their status to their partners. They further indicated that they were willing to ask their husbands if they were ready to receive the results.

"I will tell my partner. If you tested positive, you will be free, and you can even talk about your status and people will support you, but if you are not tested you are always concerned about your health." "I will tell the pastor of my church. He will pray for me. If one day I don't feel good and start thinking about bad things I will go to him to pray for me. He will support me."

In contrast, the majority of the participants who opted out were not in favour of informing their partners about the outcome of their HIV results. It would be difficult to tell their partners because of fear of being confronted with physical violence due to their HIV status. They feared of being killed and chased away.

"Some of the women are not really having a good relationship with their husbands. They are staying in one house, but everyone is doing whatever they want." HIV positive results in a relationship like this will never be accepted. A man can beat you up and tell his relatives, then the story spreads."

Another similarity that was raised by some participants who opted in and opted out was that they preferred to tell their parents, friends or family members they trusted would keep results confidential. Some preferred not to tell anybody because they did not trust anybody. Even their mothers would tell everybody about the results. They explained how difficult it is:

"I will not tell my husband, it is better to tell my parents. They will advise me on how to behave. I will not tell my partner, because he will kill me. He warned me already that I should not go for the test, otherwise he will kill me." "I will not even tell my mother that I tested positive. She will tell other people that she is not sleeping well because 'Kauna' is HIV positive. You know, most mothers can understand only if you are married because they usually blame husbands for infecting their wives." But some of us are not married, they will say: "It is your own doing because you were sleeping around and now you are sick. Just go where you got the disease from. It is better to keep quiet."

Published by Sciedu Press
In contrast, participants who opted out felt that this programme was done as kind of forcing people to be tested although they were not ready. Therefore, some participants were not sure who they would tell if they were to be tested.

“Mnh, may be I will tell my husband but I don't know that I will be tested. I just came to attend ANC and the nurses told us that we will be tested for HIV." I just came to attend ANC and the nurses told us that we will be tested for HIV. If you decline then they will refer you to one nurse who will ask why you don't want to take the test. This is kind of forcing people. But at the VCT centre, people talk to you and ask you whether you would like to take the test or not. If you don't they will leave you. Like in my case I refused, but I don't think that those who took the test will come back for their results."

\subsubsection{Reactions from the family members}

Both participants who opted in and out were concerned that the family members would react negatively. They would be blamed and discriminated against by their family members, especially the unmarried ones. Therefore, it was difficult to inform their family members because they were afraid of being stigmatized and abused. Pregnant women who opted out indicated that the unmarried women would suffer more, because they would be verbally abused by their family members and accused of being whores.

"The relationship between you and your family will break. They will stigmatize and discriminate against you. Everyone in the house will know about your status."

Participants who opted out further explained that it was high time for people not to discriminate against others because of their status. More people were infected; therefore there was no need to discriminate against each other.

"It is like that but I don't think that in the future people will continue talking or blaming others because nowadays every house is affected by this disease."

\subsection{Desired improved voluntary antenatal HIV coun- selling and testing}

Participants were requested to point out areas that need improvement.

\subsubsection{Need for education}

Some participants were not aware of the PMTCT programme, they just happened to know about it when they started the ANC services. If men were also educated, they would allow their partners to go for the test freely.

"Nurses should educate the people to let them understand the benefits of VCT. The community meetings should be for both men and women because here people are really talking 
about the advantages of the HIV test during pregnancy and in general. The men don't like to attend such meetings, they are behind. At the end of the day they will end up preventing their wives to go for the HIV test."

"People really need to be educated in order to overcome their fear. Now they are very much scared about how their husbands will react."

Participants who opted out suggested that education was needed because the information regarding the programme was not clear enough. They found that accessibility to the information of PMTCT was found to be a problematic area. They felt that community education was needed in order to know what to expect before one started with ANC.

"Health educators should inform us well, because the message is not really clear that we will be tested when we are coming to the ANC. If you are clean, it is $O K$ but if you are not then it will really bring death nearer/closer. It is very difficult to take a decision in a short time if you did not think about it before."

\subsubsection{Male involvement}

Pregnant women who opted in and out felt that the involvement of partners in the VCT was needed in order to reduce the fears among pregnant women for them to come freely for the test. They also felt that partner communication should be encouraged so that they discuss these issues in order not to blame each other.

"The pregnant women should discuss the VCT with their husbands at home and go together for testing. If that happens men will no longer blame women for the results." "It is also better to let men understand the issue of testing because they are the ones who are not allowing us as women to be tested or not to come for our results."

\subsubsection{Compulsory test}

Pregnant women who opted in suggested that it should be compulsory for all pregnant women to be tested for HIV during pregnancy in order to reduce HIV prevalence rate among children.

"All pregnant women should only be tested whether they like it or not in order for them to be helped. The time they come to deliver, they will find their results at the maternity department for them to receive the drugs. Drugs will help their babies and reduce deaths."

The group who opted in further explained that the situation will improve because currently there are many hospitals that are offering this service. In the past only bigger hospitals used to do it.

"We are happy, because, now most of the hospitals are of- fering HIV tests." Earlier, only some hospitals used to do it and people were willing to be tested but the services were not available. In some cases those who wanted had probably difficulties to travel long distances because of financial constraints.

\subsubsection{Need for the health workers to keep the information confidential}

The participants who opted out suggested that nurses should try not to reveal the HIV status of the clients. Currently, according to some of the participants, nurses seem to reveal information to the community. This makes people unwilling to take the test because the nurses will tell everybody. They further said that people who are working at the counselling office should be selected or take an oath to keep all the information confidential.

"People should not be told to disclose their status. They should not be forced to tell their families." "At counseling offices, there should be reliable people. Everything discussed should remain in the office. If it continues like, this none will use that service any more."

\section{Discussions}

In discussing these findings, it became evident that women who opted in and out demonstrated that their knowledge about the benefits of voluntary antenatal counselling and testing was almost the same. On the other hand, those who opted out just need encouragement to take the test. They do not need to fear anything, since the people infected with HIV/AIDS may remain healthy for many years even without ARV therapy, as long as they are looking after their health.

A major concern that has been identified in the literature review and confirmed by the results of the study is the negative attitudes of health workers that prevent women from taking the tests. Women were concerned that nurses would reveal their HIV status. They thought that they would be stigmatized in the community if their HIV status was revealed.

It also became evident that women were not willing to take part in the testing programme because of negative reactions from their family members. Participants explained why they would not disclose their results. They were certain that they would not get support from families regarding basic necessities such as shelter, food etcetera.

The results of the study showed the reasons pregnant women gave for not taking the test, amongst others: fear to be blamed for bringing the HIV in the family, rejection, intimidation and harassment. This led to women feeling responsible for the disease.

It was evident that women have limited control over their 
sexual activities and prevention of infections. This was indicated in the literature that women tend not to practice safe sex although they know about their status. The imbalance of power between women and their partners is what places women at risk of HIV and limits their ability to take preventive measures.

The study also revealed how some participants showed their willingness to adopt safe behaviours such as abstaining from sex during pregnancy and using of condoms. In Kenya, Kampala and Uganda, women gave the same sentiment that they went for the test in order to change their behaviour. ${ }^{[9]}$

The study results revealed that education and male involvement are needed as a way of improving the VCT services. This will help women to gain support and cooperation from their male partners. According to the findings of study done by Nashandi ${ }^{[10]}$ confirmed that the participants appeared to have adequate knowledge about the preventive measures, but without adequate personal support and cooperation from their male partners, nothing will really change. Interestingly, findings from this study showed that the majority of those who are using VCT are better educated than those not using.

\section{Conclusions}

The results of the study suggested that there was some uncertainty surrounding vertical transmission, and the women's understanding of HIV/AIDS was low. All participants should have known the causes and the preventive measures of vertical transmission. It can be concluded from the above foregoing information some participants had some knowledge on vertical transmission, but most of them, especially those who opted out did not.

\section{RECOMMENDATIONS}

The following recommendations are made based on the findings of this study:

- Stigma and discrimination has been identified as a barrier to the acceptance of voluntary antenatal counselling and testing. Therefore, there is a serious call for interventions, which deal with violence against women and men over women's sexualities in communities.

- Community education about the importance of voluntary antenatal counselling and testing and PMTCT should be strengthened. It will encourage community involvement and the reduction of stigma and discrimination in the society.

- Since the health workers are the important support system for pregnant women with HIV/AIDS, they should keep the information regarding the HIV results confidential and provide care and support to those who are HIV positive.

- Effective communication and cooperation should be encouraged between partners. It will increase the male involvement in the programme and ensure that men know the importance of voluntary antenatal counselling and testing.

- Cultural practices should be discouraged in order to prevent HIV transmission from the mother to the baby.

\section{ACKNOWLEDGeMents}

We wish to thank all the participants who took part in this study. Without their participation this study would not have been even a success or even possible. We also wish to thank the Ministry of Health and Social Services (MoHSS) for granting us permission to conduct this research study on its premises. The authors contributed as follows: Aini-Kaarin Toivo is the principal investigator, Mickey Chopra supervised the project and Daniel Opotamutale Ashipala wrote the manuscript.

\section{CONFLicts OF INTEREST Disclosure}

The researchers declare that they had no financial or personal relationship(s) which may have inappropriately influenced them in the writing of this article.

\section{REFERENCES}

[1] Government of the Republic of Namibia. Ministry of Health and Social Services. Report of the 2014 National HIV Sentinel Survey. Namibia. 2014.

[2] World Health Organization. Prevention of Mother to Child Transmission of HIV: Selection and Use of Nevirapine. Technical Notes. Geneva. WHO/HIV-AIDS/2001.3. 2001.

[3] Government of the Republic of Namibia. Ministry of Health and Social Services. Guideline for Anti-Retroviral Therapy. Namibia. 2014.

[4] Brink H. Fundamentals of Research Methodology for health Care Professionals. Second Edition. Juta \& Co, Ltd. 2014.

[5] Krueger RA. Focus groups. 2nd Edition. Thousand Oaks: Sage Publications; 2011.

[6] Patton MQ. Qualitative Evaluation and Research Methods. Thousand Oaks: Sage Publications; 2002.

[7] Health Systems Research II Reader. Postgraduate Diploma in Public Health. School of Public Health, University of Western Cape. Bellville. 2004.

[8] Krefting L. (Rigor in qualitative research. The assessment of trustworthiness. 1991; 45(3).

[9] Horizons Project/Population Councils. Prevention of Mother-to-Child HIV transmission: Assessing feasibility, acceptability and cost of services in Kenya and Zambia. HIV/AIDS operational Research. [Online]. 2003. Available from: www . popcoucil .org/horizons

[10] Nashandi JCK. Experiences and Coping strategies of Women living with HIV/AIDS: Case Study Khomas region, Namibia. Windhoek. 2002. 